\title{
Exploitation of Heterosis for Yield and Yield Contributing Character in Sesame (Sesamum indicum L.)
}

\author{
G.R. Karande ${ }^{1}$, S.V. Yamgar ${ }^{1 *}$, A.A. Waghmode ${ }^{3}$ and P.B. Wadikar ${ }^{4}$ \\ ${ }^{1}$ Department of Agricultural Botany, College of Agriculture, Latur-413512, India \\ ${ }^{2}$ Agriculture Biotechnology, Student. College of agriculture Biotchnology, Latur, India \\ ${ }^{3}$ Department of Agricultural Botany, College of Agriculture, Latur-413512 Vasantrao Naik \\ Marathwada Agricultural University, Parbhani-431 402 (M.S.), India \\ *Corresponding author
}

\begin{abstract}
A B S T R A C T
Keywords

Sesame, Heterosis,

Yield components,

Line $\mathrm{x}$ tester

analysis

Article Info

Accepted:

04 January 2018

Available Online:

10 February 2018

Present study comprised a line $\mathrm{x}$ tester analysis consists of seven female lines and three tester were crossed to estimate the heterosis for seed yield and yield contributing trait in sesame. Their 21 crosses were evaluated in two replication of randomized block design over two commercial checks Phule til and JLT-7 respectively. The morphological observations on 10 quantitative characters were recorded. The highest magnitude of heterosis over mid parent BSG-5 x LT-8 (90.44\%) and over better parent BSG-5 x LT-8 $(68.07 \%)$ for seed yield per plant. The cross BSG-5 x LT-8 (125.19\%) and the cross BSG-5 x LT-5 (109.36\%).showed the highest magnitude of standard heterosis standard check Phule til and JLT-7 respectively.
\end{abstract}

\section{Introduction}

Sesame (Sesamum indicum L.), commonly known as gingelly, til, benniseed, simsim, is a member of the order Tubiflorae and family Pedaliaceae. Sesame is highly nutritive, medicinal value, high oil (38-54\%) and protein content (18-25\%), sesame seeds are digestive, rejuvenative, anti-aging and rich in vitamins E, minerals like calcium, phosphorus, iron (white seeds), copper, magnesium, zinc and potassium. This unique composition coupled with high-unsaturated fatty acid (linolinic and tocopherol) make the sesame nearly perfect food. Its oil contains an antioxidant called sesamol which imparts a high degree of resistance against oxidative rancidity.

Sesame is one of the nine major oil seeds produced in India. The contribution of different oil seed crops to the total production has been in the order of groundnut, soybean, rapeseed, mustard, sunflower and sesame. Heterosis is a measure of deviation of progeny means from parental mean. The utilization of 
heterosis as a means of maximizing the yield of different crops has become one of the important techniques in plant breeding

\section{Materials and Methods}

The experimental material consists of three female lines viz, BSG - 1, BSG - 5, BSG - 9 and seven tester viz, IC -413197, IC- 413201, IC-413204, IC-413221, LT-4, LT-5 LT-8 were crossed in Line $\mathrm{X}$ Tester design (Kempthorne, 1957) and produced 21 hybrids in kharif, 2013 seeds were harvested separately to study heterosis in next season. The resulting 21 hybrids, 10 parents and two checks were sown in randomized block design with two replications during rabi-2013-14 at the department of Agricultural Botany, Research Farm, College of Agriculture, Latur each plot consisted of three rows of 3.0 meter length with spacing of $45 \mathrm{~cm}$ between rows and 20 $\mathrm{cm}$ within row. The recommended dose of fertilizer was applied 30:60:30 kg NPK/ha. The recommended cultural practices including plant protection measures were followed. The morphological observations on 10 quantitative characters were recorded by selecting randomly 5 competitive plants in each plot.viz., days to $50 \%$ flowering, number of branches per plant, days to maturity, plant height $(\mathrm{cm})$,number seeds per capsule, length capsule, seed yield per plant(g), 1000 seed weight(g), Oil content $(\%)$. Number of capsule per plant. The analysis was carried out in computer using software INDOSTAT as per standard procedure of heterobeltiosis, pooled values and standard heterosis at oilseed research station, Latur.

\section{Results and Discussion}

Analysis of variance for mean revealed significant differences for all the ten characters, Parents vs. hybrids interaction mean square were also significant for all the characters indicating the presence of sufficient variability in parents and hybrids. Heterosis may be positive or negative, depending on the magnitude of the hybrid means. For exploitation of hybrid vigour, high degree of heterosis for seed yield and its components is a prerequisite. The negative heterosis is important for characters like earliness. In the present investigation parent $v s$. cross interaction, mean sum of squares provided a measure of heterosis for all the characters studied. But such comparison will test only the differences between parental and hybrid group means. The significant differences could arise due to few but highly heterotic combinations (Arunachalam, 1974). Hence analysis of heterosis based on single crosses which may have no common origin has its importance. In the present investigation high magnitude of mid parent heterosis and heterobeltosis was observed for all ten characters.

The genotypes with early flowering and maturity have a special significance in multiple cropping systems. The crosses BSG-9 $x$ LT-8 (-8.22, -10.67 and -8.22) shown significantly high negative heterosis over mid parent and better parent and standard check Phule Til and JLT-7 respectively for early flowering and for days to maturity from BSG1 x IC-413221 (-3.51, -5.71, -4.07 and -4.62) would be desirable as they have shown significantly high negative heterosis over mid parent, better parent and standard check Phule Til and JLT-7 respectively. Thus it indicated that early maturity hybrids could be developed with the parental material in the present investigation. These results are in agreement with the result obtained by earlier workers Baviskar et al., (1998), Ragiba and Reddy (2000a), Rajput (2003), Thiyagu et al., (2007a) and Sundari and Kamala (2012). The high negative significant heterosis of crosses can be identified for selection for days to $50 \%$ flowering and days to maturity. For plant height the crosses BSG-1 x LT-4, BSG-1 x LT-5 and BSG-1 x LT-8 BSG-1 x IC-413221 $(-22.77,-20.24,-15.53$ and -12.34$)$ showed desirable positive heterosis over check Phule 
Til and BSG-1 x LT-4, BSG-1 x LT-5 and BSG-1 x LT-8 (-19.51, -16.86 and -11.75) for JLT-7 respectively. The heterosis for this trait was previously reported by Rajput (2003), Mothilal and Ganesan (2005) and Thiyagu et al., (2007a) also recorded high positive heterosis for this trait.

For the number of branches per plant, four crosses showed desirable positive heterosis over mid parent. High heterosis for this trait was recorded to the extent of 24.72 per cent by the cross (BSG-9 $x$ LT-5). Two crosses showed desirable positive heterosis over better parent. Heterosis over better parent was observed to the extent of 18.75 per cent by (BSG-9 x IC-413197). The crosses 9 and 15 showed desirable positive heterosis over check Phule Til and JLT-7 respectively. The heterosis for this trait was previously reported by Ragiba and Reddy (2000a), Rajput (2003), Mothilal and Ganesan (2005), Thiyagu et al., (2007a), Misra et al., (2008), Toprope (2009) and Sundari and Kamala (2012) also recorded high heterosis for this trait. For number of capsule per plant, positive heterosis is desirable. Out of 21 crosses, the crosses 19, BSG-5 x IC-413204 (101.63) and 20 crosses, BSG-5 x LT-4 (111.18) exhibited the highest significant positive heterosis over the check Phule Til and JLT-7 respectively. These results are in agreement with the result obtained by earlier workers Rajput (2003), Mothilal and Ganesan (2005), Thiyagu et al., (2007a), Misra et al., (2008) and Toprope (2009).

For number of seed per capsule, positive heterosis is desirable. Out of 21crosses, 3 crosses, BSG-1 x IC-413197 (10.81) exhibited the highest significant positive heterosis over mid parent. The crosses, BSG-1 x IC-413221 (10.54) exhibited the highest significant positive heterosis over the check Phule Til and JLT-7 respectively. These results are in agreement with the result obtained by earlier workers Baviskar et al., (1998), Ragiba and
Reddy (2000a), Rajput (2003), Mothilal and Ganesan (2005), Thiyagu et al., (2007a), Misra et al., (2008) and Toprope (2009). For length of capsule, positive heterosis is desirable. Out of 21 crosses, The cross BSG- 1 $x$ IC-413221 (6.61) exhibited the highest significant positive heterosis over mid parent. The cross BSG-5 x IC-413204 (2.64) exhibited the highest significant positive heterosis over better parent. The cross BSG-9 $x$ LT-5 (6.82) and BSG-9 x LT-5 (4.44) exhibited the highest significant positive heterosis over the check Phule Til and JLT-7 respectively. These results are in agreement with the result obtained by earlier workers Navodiya et al., (1995), Baviskar et al., (1998), Ragiba and Reddy (2000a) and Rajput (2003). For 1000 seed weight, Among the 21 crosses, 15 and 13 crosses showed significant positive heterosis over mid parent and better parent respectively. The cross BSG-5 x LT-5 (81.18) exhibited the highest significant positive heterosis over mid parent. The cross BSG-5 x LT-5 (79.07) exhibited the highest significant positive heterosis over better parent. The cross BSG-5 x LT-5 (13.24) exhibited the highest positive heterosis over the check Phule Til. The heterosis for this trait was previously reported by Rajput (2003), Mothilal and Ganesan (2005), Thiyagu et al., (2007a), Misra et al., (2008) and Sundari and Kamala (2012). For oil content, Among the 21 crosses, 2, 14 and 16 crosses showed significant positive heterosis over mid parent, check Phule Til and JLT-7 respectively. The cross BSG-1 x IC-413197 (10.31) exhibited the highest significant positive heterosis over mid parent. The cross BSG-9 x LT-4 (19.07) and BSG-9 x LT-4 (24.23) exhibited the highest significant positive heterosis over the check Phule Til and JLT-7 respectively. These results are in agreement with the result obtained by earlier workers Rajput (2003) and Thiyagu et al., (2007a). 
Table.1 Mean performance of parents and crosses for 10 characters in sesame

\begin{tabular}{|c|c|c|c|c|c|c|c|c|c|c|c|}
\hline Sr. No. & Characters & $\begin{array}{l}\text { Days to } \mathbf{5 0 \%} \\
\text { flowering }\end{array}$ & $\begin{array}{ll}\text { Day } & \text { to } \\
\text { maturity } & \end{array}$ & $\begin{array}{l}\text { Plant } \\
\text { height } \\
(\mathrm{cm})\end{array}$ & $\begin{array}{l}\text { No. of } \\
\text { branches } \\
\text { / plant }\end{array}$ & $\begin{array}{l}\text { No. of } \\
\text { capsule } / \\
\text { plant }\end{array}$ & $\begin{array}{l}\text { No. of } \\
\text { seed / } \\
\text { capsule }\end{array}$ & $\begin{array}{l}\text { Length } \\
\text { of capsule } \\
\text { (cm) }\end{array}$ & $\begin{array}{l}1000 \text { Seed } \\
\text { weight } \\
\text { (gm) }\end{array}$ & $\begin{array}{l}\text { Oil } \\
\text { content } \\
(\%)\end{array}$ & $\begin{array}{l}\text { Seed yield } \\
\text { plant } \\
\text { (gm) }\end{array}$ \\
\hline \multicolumn{12}{|c|}{ Lines } \\
\hline 1 & BSG-1 & 38 & 84 & 67 & 4.9 & 53 & 60 & 2.29 & 2.25 & 41.9 & 7.1 \\
\hline 2 & BSG-5 & 37 & 86 & 82 & 4.2 & 86 & 59 & 2.25 & 2.10 & 48.8 & 10.6 \\
\hline 3 & BSG-9 & 36 & 83 & 74 & 4.8 & 41 & 56 & 2.225 & 2.20 & 47.2 & 5.0 \\
\hline \multicolumn{12}{|c|}{ Testers } \\
\hline 4 & IC-413197 & 36 & 83 & 88 & 4.75 & 44 & 48 & 2.24 & 2.20 & 41.5 & 4.5 \\
\hline 5 & IC-413201 & 35 & 84 & 86 & 4.8 & 45 & 57 & 2.36 & 2.20 & 46.6 & 5.6 \\
\hline 6 & IC-413204 & 34 & 85 & 87 & 4.25 & 67 & 53 & 2.27 & 2.20 & 44.1 & 7.9 \\
\hline 7 & IC-413221 & 35 & 88 & 77 & 4 & 71 & 63 & 2.10 & 2.20 & 43.7 & 9.8 \\
\hline 8 & LT-4 & 37 & 87 & 67 & 4.45 & 75 & 59 & 2.15 & 2.20 & 44.3 & 9.6 \\
\hline 9 & LT-5 & 37 & 84 & 67 & 4.1 & 71 & 49 & 2.34 & 2.15 & 44.7 & 7.5 \\
\hline 10 & LT-8 & 38 & 84 & 76 & 4.8 & 66 & 58 & 2.18 & 2.15 & 44 & 8.1 \\
\hline \multicolumn{12}{|c|}{ Crosses } \\
\hline 11 & BSG-1 X IC-413197 & 35 & 83 & 80 & 5.1 & 50 & 60 & 2.35 & 3.05 & 46 & 9.1 \\
\hline 12 & BSG-1 X IC-413201 & 34 & 82 & 81 & 5.25 & 52 & 61 & 2.34 & 3.25 & 48.5 & 10.1 \\
\hline 13 & BSG-1 X IC-413204 & 34 & 83 & 83 & 4.95 & 64 & 60 & 2.29 & 3.55 & 41.9 & 13.4 \\
\hline 14 & BSG-1 X IC-413221 & 35 & 83 & 78 & 5 & 65 & 65 & 2.34 & 3.20 & 46.1 & 13.5 \\
\hline 15 & BSG-1 X LT-4 & 35 & 84 & 67 & 4.65 & 64 & 60 & 2.32 & 3.50 & 40.7 & 13.3 \\
\hline 16 & BSG-1 X LT-5 & 39 & 82 & 71 & 5.55 & 61 & 59 & 2.33 & 2.60 & 40.9 & 9.2 \\
\hline 17 & BSG-1 X LT-8 & 38 & 84 & 75 & 5.1 & 65 & 60 & 2.28 & 3.50 & 46.4 & 13.5 \\
\hline 18 & BSG-5 X IC-413197 & 34 & 83 & 90 & 4.75 & 71 & 57 & 2.24 & 3 & 45.9 & 12 \\
\hline 19 & BSG-5 X IC-413201 & 34 & 84 & 88 & 4.95 & 70 & 58 & 2.30 & 3.40 & 46.20 & 13.7 \\
\hline 20 & BSG-5 X IC-413204 & 34 & 84 & 86 & 4.30 & 80 & 58 & 2.33 & 3.25 & 47.5 & 15.1 \\
\hline 21 & BSG-5 X IC-413221 & 34 & 85 & 85 & 4.10 & 80 & 63 & 2.19 & 3.05 & 44.2 & 15.3 \\
\hline 22 & BSG-5 X LT-4 & 34 & 87 & 80 & 4.65 & 80 & 60 & 2.26 & 2.85 & 47 & 13.6 \\
\hline 23 & BSG-5 X LT-5 & 35 & 84 & 80 & 4.30 & 80 & 54 & 2.34 & 3.85 & 42.7 & 16.6 \\
\hline 24 & BSG-5 X LT-8 & 38 & 85 & 80 & 5.35 & 80 & 64 & 2.24 & 3.50 & 47.7 & 17.9 \\
\hline 25 & BSG-9 X IC-413197 & 36 & 81 & 85 & 5.70 & 48 & 56 & 2.23 & 2.10 & 46.7 & 5.5 \\
\hline 26 & BSG-9 X IC-413201 & 34 & 82 & 82 & 5.15 & 48 & 62 & 2.29 & 2.95 & 46.3 & 8.7 \\
\hline 27 & BSG-9 X IC-413204 & 34 & 82 & 86 & 4.90 & 55 & 54 & 2.30 & 3.40 & 46.3 & 10.2 \\
\hline 28 & BSG-9 X IC-413221 & 34 & 83 & 79 & 4.80 & 61 & 63 & 2.21 & 2.10 & 41.2 & 8 \\
\hline 29 & BSG-9 X LT-4 & 34 & 84 & 70 & 4.65 & 62 & 60 & 2.22 & 2.35 & 48.7 & 8.5 \\
\hline 30 & BSG-9 X LT-5 & 35 & 84 & 70 & 5.55 & 61 & 52 & 2.35 & 2.60 & 48 & 8 \\
\hline 31 & BSG-9 X LT-8 & 34 & 82 & 79 & 5.25 & 55 & 63 & 2.20 & 2.80 & 44.8 & 9.4 \\
\hline \multicolumn{12}{|c|}{ Checks } \\
\hline & JLT- 7 & 37 & 87 & 85 & 4 & 38 & 59 & 2.25 & 3.5 & 39.2 & 8.9 \\
\hline & Phule Til & 37 & 86 & 89 & 4.3 & 40 & 59 & 2.20 & 3.4 & 40.9 & 7.9 \\
\hline & SE $( \pm)$ & 1.01 & 1.10 & 3.60 & 0.23 & 4.09 & 2.10 & 0.03 & 0.24 & 1.49 & 1.06 \\
\hline & CD \% & 2.92 & 3.19 & 10.38 & 0.67 & 11.80 & 6.07 & 0.10 & 0.71 & 4.31 & 3.05 \\
\hline
\end{tabular}


Table.2 Estimates of heterosis in percentage over mid parent (M.P.), better parent (B.P.) and standard check (S.C.) for yield and yield contributing characters in sesame

\begin{tabular}{|c|c|c|c|c|c|c|c|c|c|}
\hline \multirow{2}{*}{$\begin{array}{c}\text { Sr } \\
\text { No. }\end{array}$} & \multirow[t]{2}{*}{ Name of crosses } & \multicolumn{4}{|c|}{ Days to $50 \%$ flowering } & \multicolumn{4}{|c|}{ Days to maturity } \\
\hline & & MP & BP & SC-1 & $\mathrm{SC}-2$ & MP & BP & SC-1 & SC-2 \\
\hline & & & & Phule Til & JLT-7 & & & Phule Til & JLT-7 \\
\hline 1 & BSG-1 X IC-413197 & -4.11 & -6.67 & -4.11 & -4.11 & -0.60 & -1.20 & $-4.07 *$ & $-4.62 *$ \\
\hline 2 & BSG-1 X IC-413201 & $-6.94 *$ & $-10.67 * *$ & $-8.22 *$ & $-8.22 *$ & -2.40 & -2.40 & $-5.23 * *$ & $-5.78 * *$ \\
\hline 3 & BSG-1 X IC-413204 & -4.90 & $-9.33^{*}$ & -6.85 & -6.85 & -1.19 & -1.78 & -3.49 & $-4.05^{*}$ \\
\hline 4 & BSG-1 X IC-413221 & -2.78 & -6.67 & -4.11 & -4.11 & $-3.51 *$ & $-5.71 * *$ & $-4.07 *$ & $-4.62 *$ \\
\hline 5 & BSG-1 X LT-4 & -5.41 & -6.67 & -4.11 & -4.11 & -1.47 & -3.45 & -2.33 & -2.89 \\
\hline 6 & BSG-1 X LT-5 & 4.05 & 2.67 & 5.48 & 5.48 & -1.80 & -1.80 & $-4.65^{*}$ & $-5.20 * *$ \\
\hline 7 & BSG-1 X LT-8 & 0.00 & 0.00 & 2.74 & 2.74 & 0.00 & 0.00 & -2.91 & -3.47 \\
\hline 8 & BSG-5 X IC-413197 & $-6.94 *$ & $-8.22 *$ & $-8.22 *$ & $-8.22 *$ & -2.08 & $-4.07 *$ & $-4.07 *$ & $-4.62 *$ \\
\hline 9 & BSG-5 X IC-413201 & -5.63 & $-8.22 *$ & $-8.22 *$ & $-8.22 *$ & -1.47 & -2.91 & -2.91 & -3.47 \\
\hline 10 & BSG-5 X IC-413204 & -4.96 & $-8.22 *$ & $-8.22 *$ & $-8.22 *$ & -1.47 & -2.33 & -2.33 & -2.89 \\
\hline 11 & BSG-5 X IC-413221 & -5.63 & $-8.22 *$ & $-8.22 *$ & $-8.22 *$ & -2.02 & -2.86 & -1.16 & -1.73 \\
\hline 12 & BSG-5 X LT-4 & $-6.85^{*}$ & -6.85 & -6.85 & -6.85 & 0.00 & -0.57 & 0.58 & 0.00 \\
\hline 13 & BSG-5 X LT-5 & -5.48 & -5.48 & -5.48 & -5.48 & -0.88 & -2.33 & -2.33 & -2.89 \\
\hline 14 & BSG-5 X LT-8 & 2.70 & 1.33 & 4.11 & 4.11 & -0.29 & -1.74 & -1.74 & -2.31 \\
\hline 15 & BSG-9 X IC-413197 & 0.00 & 0.00 & -2.74 & -2.74 & -1.82 & -1.82 & $-5.81 * *$ & $-6.36 * *$ \\
\hline 16 & BSG-9 X IC-413201 & -4.29 & -5.63 & $-8.22 *$ & $-8.22 *$ & -1.81 & -2.40 & $-5.23 * *$ & $-5.78 * *$ \\
\hline 17 & BSG-9 X IC-413204 & -3.60 & -5.63 & $-8.22 *$ & $-8.22 *$ & -1.80 & -2.96 & $-4.65^{*}$ & $-5.20 * *$ \\
\hline 18 & BSG-9 X IC-413221 & -4.29 & -5.63 & $-8.22 *$ & $-8.22 *$ & -2.35 & $-5.14 * *$ & -3.49 & $-4.05^{*}$ \\
\hline 19 & BSG-9 X LT-4 & -5.56 & -6.85 & -6.85 & -6.85 & -1.47 & $-4.02 *$ & -2.91 & -3.47 \\
\hline 20 & BSG-9 X LT-5 & -4.17 & -5.48 & -5.48 & -5.48 & 1.20 & 0.60 & -2.33 & -2.89 \\
\hline 21 & BSG-9 X LT-8 & $-8.22 *$ & $-10.67 * *$ & $-8.22 *$ & $-8.22 *$ & -1.20 & -1.80 & $-4.65^{*}$ & $-5.20 * *$ \\
\hline & SE $( \pm)$ & 1.187 & 1.371 & 1.371 & 1.371 & 1.329 & 1.534 & 1.534 & 1.534 \\
\hline
\end{tabular}


Continue ...

\begin{tabular}{|c|c|c|c|c|c|c|c|c|c|}
\hline \multirow{2}{*}{$\begin{array}{l}\text { Sr } \\
\text { No. }\end{array}$} & \multirow[t]{2}{*}{ Name of crosses } & \multicolumn{4}{|c|}{ Plant height (cm) } & \multicolumn{4}{|c|}{ No. of branches / plant } \\
\hline & & MP & BP & SC-1 & SC-2 & MP & BP & SC-1 & SC-2 \\
\hline & & & & Phule Til & JLT-7 & & & Phule Til & JLT-7 \\
\hline 1 & BSG-1 X IC-413197 & 3.53 & -8.83 & -9.81 & -5.99 & 5.70 & 4.08 & $18.60 *$ & $27.50 * *$ \\
\hline 2 & BSG-1 X IC-413201 & 5.52 & -6.38 & -8.96 & -5.11 & 8.25 & 7.14 & $22.09 *$ & $31.25 * *$ \\
\hline 3 & BSG-1 X IC-413204 & 7.00 & -5.61 & -6.99 & -3.06 & 8.20 & 1.02 & 15.12 & $23.75^{*}$ \\
\hline 4 & BSG-1 X IC-413221 & 8.29 & 1.24 & $-12.34 *$ & -8.64 & 12.36 & 2.04 & $16.28 *$ & $25.00 * *$ \\
\hline 5 & BSG-1 X LT-4 & 2.39 & 2.24 & $-22.77 * *$ & $-19.51 * *$ & -0.53 & -5.10 & 8.14 & 16.25 \\
\hline 6 & BSG-1 X LT-5 & 5.40 & 4.89 & $-20.24 * *$ & $-16.86^{* *}$ & $23.33 * *$ & 13.27 & $29.07 * *$ & $38.75 * *$ \\
\hline 7 & BSG-1 X LT-8 & 5.55 & -0.53 & $-15.33 * *$ & $-11.75^{*}$ & 5.15 & 4.08 & $18.60 *$ & $27.50 * *$ \\
\hline 8 & BSG-5 X IC-413197 & 5.91 & 2.56 & 1.47 & 5.76 & 6.15 & 0.00 & 10.47 & $18.75 *$ \\
\hline 9 & BSG-5 X IC-413201 & 3.89 & 1.45 & -1.35 & 2.82 & 10.00 & 3.12 & 15.12 & $23.75^{*}$ \\
\hline 10 & BSG-5 X IC-413204 & 0.83 & -2.17 & -3.61 & 0.47 & 1.78 & 1.18 & 0.00 & 7.50 \\
\hline 11 & BSG-5 X IC-413221 & 7.23 & 3.71 & -3.89 & 0.18 & 0.00 & -2.38 & -4.65 & 2.50 \\
\hline 12 & BSG-5 X LT-4 & 7.24 & -2.68 & -9.81 & -5.99 & 7.51 & 4.49 & 8.14 & 16.25 \\
\hline 13 & BSG-5 X LT-5 & 7.25 & -2.37 & -9.53 & -5.70 & 3.61 & 2.38 & 0.00 & 7.50 \\
\hline 14 & BSG-5 X LT-8 & 0.82 & -3.28 & $-10.37 *$ & -6.58 & $18.89 * *$ & 11.46 & $24.42 * *$ & $33.75 * *$ \\
\hline 15 & BSG-9 X IC-413197 & 4.68 & -3.42 & -4.45 & -0.41 & $19.37 * *$ & $18.75^{*}$ & $32.56^{* *}$ & $42.50 * *$ \\
\hline 16 & BSG-9 X IC-413201 & 1.80 & -5.33 & -7.95 & -4.05 & 7.29 & 7.29 & $19.77 *$ & $28.75 * *$ \\
\hline 17 & BSG-9 X IC-413204 & 6.02 & -2.00 & -3.44 & 0.65 & 8.29 & 2.08 & 13.95 & $22.50 *$ \\
\hline 18 & BSG-9 X IC-413221 & 4.66 & 2.86 & $-10.94 *$ & -7.17 & 9.09 & 0.00 & 11.63 & $20.00 *$ \\
\hline 19 & BSG-9 X LT-4 & -0.83 & -5.62 & $-21.08 * *$ & $-17.74 * *$ & 0.54 & -3.13 & 8.14 & 16.25 \\
\hline 20 & BSG-9 X LT-5 & -1.50 & -5.96 & $-21.36^{* *}$ & $-18.04 * *$ & $24.72 * *$ & $15.62 *$ & $29.07 * *$ & $38.75 * *$ \\
\hline 21 & BSG-9 X LT-8 & 5.23 & 4.30 & -11.22 & -7.46 & 9.38 & 9.37 & $22.09 *$ & $31.25 * *$ \\
\hline & SE $( \pm)$ & 3.753 & 4.333 & 4.333 & 4.333 & 0.289 & 0.334 & 0.334 & 0.334 \\
\hline
\end{tabular}


Continue ......

\begin{tabular}{|c|c|c|c|c|c|c|c|c|c|}
\hline \multirow{2}{*}{$\begin{array}{l}\text { Sr } \\
\text { No. }\end{array}$} & \multirow[t]{2}{*}{ Name of crosses } & \multicolumn{4}{|c|}{ No. of capsule / plant } & \multicolumn{4}{|c|}{ No. of seed / capsule } \\
\hline & & MP & BP & SC-1 & SC-2 & MP & BP & SC-1 & SC-2 \\
\hline & & & & Phule Til & JLT-7 & & & Phule Til & JLT-7 \\
\hline 1 & BSG-1 X IC-413197 & 4.00 & -5.12 & $25.75^{*}$ & $31.71 *$ & $10.81 *$ & -0.42 & 1.96 & 1.96 \\
\hline 2 & BSG-1 X IC-413201 & 6.10 & -1.90 & $30.03 *$ & $36.18 * *$ & 3.49 & 1.00 & 3.40 & 3.40 \\
\hline 3 & BSG-1 X IC-413204 & 6.73 & -4.49 & $60.30 * *$ & $67.89 * *$ & 5.20 & -0.83 & 1.53 & 1.53 \\
\hline 4 & BSG-1 X IC-413221 & 5.04 & -8.57 & $63.57 * *$ & $71.32 * *$ & 5.69 & 3.50 & $10.54 *$ & $10.54^{*}$ \\
\hline 5 & BSG-1 X LT-4 & 0.04 & $-14.56^{*}$ & $59.92 * *$ & $67.50 * *$ & 1.43 & 0.17 & 2.55 & 2.55 \\
\hline 6 & BSG-1 X LT-5 & -1.58 & $-14.17 *$ & $52.89 * *$ & $60.13 * *$ & 6.95 & -2.82 & -0.51 & -0.51 \\
\hline 7 & BSG-1 X LT-8 & 9.57 & -1.29 & $63.19 * *$ & $70.92 * *$ & 1.53 & -0.58 & 1.79 & 1.79 \\
\hline 8 & BSG-5 X IC-413197 & 9.01 & $-18.02 * *$ & $77.76 * *$ & $86.18 * *$ & 6.74 & -3.06 & -3.06 & -3.06 \\
\hline 9 & BSG-5 X IC-413201 & 6.10 & $-19.41 * *$ & $74.75^{* *}$ & $83.03 * *$ & 0.17 & -1.11 & -1.11 & -1.11 \\
\hline 10 & BSG-5 X IC-413204 & 4.83 & -7.01 & $101.63 * *$ & $111.18 * *$ & 3.66 & -1.19 & -1.19 & -1.19 \\
\hline 11 & BSG-5 X IC-413221 & 1.27 & -7.59 & $100.38 * *$ & $109.87 * *$ & 4.11 & 0.80 & 7.65 & 7.65 \\
\hline 12 & BSG-5 X LT-4 & -0.19 & -7.01 & $101.63 * *$ & $111.18 * *$ & 1.53 & 1.45 & 1.45 & 1.45 \\
\hline 13 & BSG-5 X LT-5 & 1.97 & -7.13 & $101.38 * *$ & $110.92 * *$ & 0.09 & -8.08 & -8.08 & -8.08 \\
\hline 14 & BSG-5 X LT-8 & 4.60 & -7.82 & $99.87 * *$ & $109.34 * *$ & $10.56^{*}$ & 9.52 & 9.52 & 9.52 \\
\hline 15 & BSG-9 X IC-413197 & 13.02 & 9.77 & 19.97 & 25.66 & 7.42 & -0.09 & -5.19 & -5.19 \\
\hline 16 & BSG-9 X IC-413201 & 12.82 & 8.04 & 21.61 & $27.37 *$ & 8.84 & 7.42 & 4.68 & 4.68 \\
\hline 17 & BSG-9 X IC-413204 & 2.32 & $-17.44 *$ & $38.57 * *$ & $45.13 * *$ & -1.19 & -3.41 & -8.33 & -8.33 \\
\hline 18 & BSG-9 X IC-413221 & 9.18 & -13.97 & $53.89 * *$ & $61.18 * *$ & 5.90 & 0.00 & 6.80 & 6.80 \\
\hline 19 & BSG-9 X LT-4 & 6.67 & $-17.32 *$ & $54.77 * *$ & $62.11 * *$ & 4.10 & 1.53 & 1.36 & 1.36 \\
\hline 20 & BSG-9 X LT-5 & 8.13 & $-14.67 *$ & $52.01 * *$ & $59.21 * *$ & -0.95 & -6.81 & $-11.56^{*}$ & $-11.56^{*}$ \\
\hline 21 & BSG-9 X LT-8 & 2.06 & $-17.17 *$ & $36.93 * *$ & $43.42 * *$ & $10.57^{*}$ & 8.75 & 6.72 & 6.72 \\
\hline & SE $( \pm)$ & 4.152 & 4.795 & 4.795 & 4.795 & 2.568 & 2.966 & 2.966 & 2.966 \\
\hline
\end{tabular}


Continue ...

\begin{tabular}{|c|c|c|c|c|c|c|c|c|c|}
\hline \multirow{2}{*}{$\begin{array}{l}\text { Sr } \\
\text { No. }\end{array}$} & \multirow[t]{2}{*}{ Name of crosses } & \multicolumn{4}{|c|}{ Length of capsule / plant (cm) } & \multicolumn{4}{|c|}{1000 Seed weight (gm) } \\
\hline & & MP & BP & $\mathrm{SC}-1$ & SC-2 & MP & BP & SC-1 & SC-2 \\
\hline & & & & Phule Til & JLT-7 & & & Phule Til & JLT-7 \\
\hline 1 & BSG-1 X IC-413197 & 3.75 & 2.62 & $6.82 * *$ & 4.44 & $37.08 *$ & $35.56^{*}$ & -10.29 & -12.86 \\
\hline 2 & BSG-1 X IC-413201 & 0.65 & -0.85 & $6.36^{*}$ & 4.00 & $46.07 * *$ & $44.44 *$ & -4.41 & -7.14 \\
\hline 3 & BSG-1 X IC-413204 & 0.44 & 0.00 & 4.09 & 1.78 & $59.55 * *$ & $57.78 * *$ & 4.41 & 1.43 \\
\hline 4 & BSG-1 X IC-413221 & $6.61 * *$ & 2.18 & $6.36^{*}$ & 4.00 & $43.82 * *$ & $42.22 *$ & -5.18 & -8.57 \\
\hline 5 & BSG-1 X LT-4 & $4.73 *$ & 1.53 & $5.68^{*}$ & 3.33 & $57.30 * *$ & $55.56^{* *}$ & 2.94 & 0.00 \\
\hline 6 & BSG-1 X LT-5 & 0.86 & -0.21 & $6.14^{*}$ & 3.78 & 18.18 & 15.56 & $-23.53^{*}$ & $-25.71^{*}$ \\
\hline 7 & BSG-1 X LT-8 & 2.24 & -0.22 & 3.86 & 1.56 & $59.09 * *$ & $55.56^{* *}$ & 2.94 & 0.00 \\
\hline 8 & BSG-5 X IC-413197 & 0.00 & -0.22 & 2.05 & -0.22 & $39.53 *$ & $36.36^{*}$ & -11.76 & -14.29 \\
\hline 9 & BSG-5 X IC-413201 & 0.00 & -2.33 & $4.77^{*}$ & 2.44 & $58.14 * *$ & $54.55^{* *}$ & 0.00 & -2.86 \\
\hline 10 & BSG-5 X IC-413204 & 3.10 & 2.64 & $5.91^{*}$ & 3.56 & $51.16^{* *}$ & $47.73^{* *}$ & -4.41 & -7.14 \\
\hline 11 & BSG-5 X IC-413221 & 0.69 & -2.67 & -0.45 & -2.67 & $41.86^{* *}$ & $38.64 *$ & -10.29 & -12.86 \\
\hline 12 & BSG-5 X LT-4 & 2.73 & 0.44 & 2.73 & 0.44 & $32.56^{*}$ & 29.55 & -16.18 & -18.57 \\
\hline 13 & BSG-5 X LT-5 & 2.18 & 0.21 & $6.59 * *$ & 4.22 & $81.18^{* *}$ & $79.07 * *$ & 13.24 & 10.00 \\
\hline 14 & BSG-5 X LT-8 & 1.13 & -0.44 & 1.82 & -0.44 & $64.71 * *$ & $62.79 * *$ & 2.94 & 0.00 \\
\hline 15 & BSG-9 X IC-413197 & 0.11 & -0.22 & 1.59 & -0.67 & -4.55 & -4.55 & $-38.24 * *$ & $-40.00 *$ \\
\hline 16 & BSG-9 X IC-413201 & -0.11 & -2.97 & 4.09 & 1.78 & $34.09 *$ & 34.09 & -13.24 & -15.71 \\
\hline 17 & BSG-9 X IC-413204 & 2.56 & 1.54 & $4.77 *$ & 2.44 & $54.55^{* *}$ & $54.55^{* *}$ & 0.00 & -2.86 \\
\hline 18 & BSG-9 X IC-413221 & 2.20 & -0.67 & 0.45 & -1.78 & -4.55 & -4.55 & $-38.24 * *$ & $-40.00 * *$ \\
\hline 19 & BSG-9 X LT-4 & 1.71 & 0.00 & 1.14 & -1.11 & 6.82 & 6.82 & $-30.88 * *$ & $-32.86^{* *}$ \\
\hline 20 & BSG-9 X LT-5 & 2.96 & 0.43 & $6.82 * *$ & 4.44 & 19.54 & 18.18 & $-23.53^{*}$ & $-25.71 *$ \\
\hline 21 & BSG-9 X LT-8 & -0.11 & -1.12 & 0.00 & -2.22 & 28.74 & 27.27 & -17.65 & -20.00 \\
\hline & SE $( \pm)$ & 0.043 & 0.049 & 0.049 & 0.049 & 0.313 & 0.362 & 0.362 & 0.362 \\
\hline
\end{tabular}

*and $* *$ indicated significance at 5 and 1 per cent level respectively 
Continue ......

\begin{tabular}{|c|c|c|c|c|c|c|c|c|c|}
\hline \multirow{2}{*}{$\begin{array}{c}\text { Sr } \\
\text { No. }\end{array}$} & \multirow[t]{2}{*}{ Name of crosses } & \multicolumn{4}{|c|}{ Oil content $(\%)$} & \multicolumn{4}{|c|}{ Seed yield / plant (gm) } \\
\hline & & MP & BP & SC-1 & SC-2 & MP & $\mathbf{B P}$ & SC-1 & SC-2 \\
\hline & & & & Phule Til & JLT-7 & & & Phule Til & JLT-7 \\
\hline 1 & BSG-1 X IC-413197 & $10.31 *$ & 9.79 & $12.47 *$ & $17.35 * *$ & $55.57 *$ & 27.82 & 14.57 & 2.24 \\
\hline 2 & BSG-1 X IC-413201 & $9.60 *$ & 4.08 & $18.58 * *$ & $23.72 * *$ & $58.78 * *$ & 42.26 & 27.51 & 13.79 \\
\hline 3 & BSG-1 X IC-413204 & -2.56 & -4.99 & 2.44 & 6.89 & $78.52 * *$ & $69.83 * *$ & $68.66 * *$ & $50.50 * *$ \\
\hline 4 & BSG-1 X IC-413221 & 7.71 & 5.49 & $12.71 *$ & $17.60 * *$ & $58.78 * *$ & $36.63 *$ & $69.85 * *$ & $51.57 * *$ \\
\hline 5 & BSG-1 X LT-4 & -5.57 & -8.13 & -0.49 & 3.83 & $59.36 * *$ & $38.77 *$ & $67.71 * *$ & $49.66 * *$ \\
\hline 6 & BSG-1 X LT-5 & -5.54 & -8.50 & 0.00 & 4.34 & 26.89 & 23.80 & 16.65 & 4.09 \\
\hline 7 & BSG-1 X LT-8 & 8.03 & 5.45 & $13.45^{*}$ & $18.37 * *$ & $77.31 * *$ & $66.18 * *$ & $70.35 * *$ & $52,02 * *$ \\
\hline 8 & BSG-5 X IC-413197 & 1.66 & -5.94 & $12.22 *$ & $17.09 * *$ & $58.37 * *$ & 13.27 & $51.76^{*}$ & $35.43^{*}$ \\
\hline 9 & BSG-5 X IC-413201 & -3.14 & -5.33 & $12.96^{*}$ & $17.86^{* *}$ & $67.94 * *$ & 28.46 & $72.11 * *$ & $53.59 * *$ \\
\hline 10 & BSG-5 X IC-413204 & 2.26 & -2.66 & $16.14 * *$ & $21.17 * *$ & $62.84 * *$ & $41.77 * *$ & $89.95 * *$ & $69.51 * *$ \\
\hline 11 & BSG-5 X IC-413221 & -4.43 & $-9.43 *$ & 8.07 & $12.76^{*}$ & $49.61 * *$ & $44.21 * *$ & $93.22 * *$ & $72.42 * *$ \\
\hline 12 & BSG-5 X LT-4 & 0.97 & -3.69 & $14.91 *$ & $19.90 * *$ & $34.53^{*}$ & 27.94 & $71.42 * *$ & $52.97 * *$ \\
\hline 13 & BSG-5 X LT-5 & $-8.66^{*}$ & $-12.50^{*}$ & 4.40 & 8.93 & $83.48 * *$ & $56.26^{* *}$ & $109.36^{* *}$ & $86.83 * *$ \\
\hline 14 & BSG-5 X LT-8 & 2.80 & -2.25 & $16.63 * *$ & $21.68 * *$ & $90.44 * *$ & $68.07 * *$ & $125.19 * *$ & $100.95^{* *}$ \\
\hline 15 & BSG-9 X IC-413197 & 5.30 & -1.06 & $14.18^{*}$ & $19.13 * *$ & 16.28 & 11.24 & -29.77 & $-37.33^{*}$ \\
\hline 16 & BSG-9 X IC-413201 & -1.28 & -1.91 & $13.20 *$ & $18.11 * *$ & $64.59 *$ & $55.49 *$ & 10.36 & -1.51 \\
\hline 17 & BSG-9 X IC-413204 & 1.42 & -1.91 & $13.20^{*}$ & $18.11 * *$ & $58.62 * *$ & 29.73 & 28.83 & 14.97 \\
\hline 18 & BSG-9 X IC-413221 & $-9.35^{*}$ & $-12.71 *$ & 0.73 & 5.10 & 8.31 & -18.34 & 1.51 & -9.42 \\
\hline 19 & BSG-9 X LT-4 & 6.45 & 3.18 & $19.07 * *$ & $24.23 * *$ & 16.42 & -11.38 & 7.10 & -4.43 \\
\hline 20 & BSG-9 X LT-5 & 4.46 & 1.69 & $17.36^{* *}$ & $22.45^{* *}$ & 28.78 & 7.53 & 1.32 & -9.59 \\
\hline 21 & BSG-9 X LT-8 & -1.75 & -5.08 & 9.54 & $14.29^{*}$ & $43.50 *$ & 15.93 & 18.84 & 6.05 \\
\hline & $\mathrm{SE}( \pm)$ & 1.876 & 2.166 & 2.166 & 2.166 & 1.268 & 1.464 & 1.464 & 1.464 \\
\hline
\end{tabular}


In the present investigation highest magnitude of heterosis was observed for seed yield per plant. Heterosis over mid parent was the exhibited the highest significant positive of 90.44 per cent (BSG-5 x LT-8) and heterobeltosis to the of 69.83 per cent (BSG-1 $\mathrm{x}$ IC-413204). The cross BSG-5 x LT-8 (125.19) and BSG-5 x LT-8 (100.95) exhibited the highest significant positive heterosis over the check Phule Til and JLT-7 respectively. Out of 21 crosses, 16 crosses showed significant positive heterosis over mid parent, 9 over better parent, 11 and 12 over standard check Phule Til and JLT-7. These results are in agreement with the results of Ragiba and Reddy (2000a), Rajput (2003), Mothilal and Ganesan (2005), Thiyagu et al., (2007a), Misra et al., (2008), Toprope (2009), Georgiev et al., (2011) and Sundari and Kamala (2012) earlier workers who also observed higher magnitude of heterosis for this trait (Table 1 and 2).

Analysis of variance for mean revealed significant differences for all the ten characters studied, Parents vs. hybrids interaction mean square were also significant for all the characters indicating the presence of sufficient variability in parents and hybrids. The highest magnitude of heterosis over mid parent BSG-5 x LT-8 (90.44\%) followed by BSG-5 x LT-5 $(83.48 \%)$ and over better parent BSG-5 x LT-8 $(68.07 \%)$ followed by BSG-5 x LT-5 (56.26\%) for seed yield per plant. The cross BSG-5 x LT$8(125.19 \%)$ showed the highest magnitude of standard heterosis followed by the cross BSG-5 x LT-5 (109.36\%). And (100.95) and (86.83) standared check Phule til and JLT-7 respectively.

\section{References}

Baviskar, A. P.; Y. M. Shinde; P. H. Bhade and G. C. Shinde. 1998. Heterosis in sesame and its relationship with combining ability. $J$.
Maharashtra Agric. Univ., 23 (1): 65 - 66. Journal of Plant Breeding 1: 33 - 36.

Chavan, A. A.; V. G. Manke and P. R. Chopade. 1982. Components of Heterosis and inbreeding depression studies in sesame (Sesamum indicum L.). J. Maharashtra agric. Univ. 7 (1):15 - 16.

Dixit, R. K. 1976a. Heterosis and inbreeding depression in sesame. Indian J. agric. Sci. 46 (4): $514-517$.

Dusane, S. M.; U. S. Surve and R. G. Rodge. 2001. Extent of useful Heterosis for yield attributes in sesame, Res. Crops. 2 (3): 457 $-459$.

Misra, R. C., Ch. H. P. Mishra, P. K. Sahu and P. K. Das. 2008. Heterosis and its relationship with combining ability, parental diversity and per se performance in sesame. Agric. Sci. Digest, 28 (4): 254- 257.

Mothilal, A. and K. N. Ganesan. 2005. Heterosis studies in sesame (Sesamum indicum L.) Agric. Sci. Digest, 25 (1): 74 - 76.

Navodiya, L. J.; P. R. Godhani and R. S. Fougat. 1995. Heterosis in sesame. Guj. agric. Univ. J. 20 (2) : 73 -77.

Ragiba, M. and C. R. Reddy. 2000a. Heterosis and inbreeding depression in sesame (Sesamum indicum L.). Ann. Agric. Res. 21 (1): 338 341.

Reddy, C. D. R and S. Haripriya. 1990. Genetic architecture, combining ability and heterosis for certain physiological parameters in sesame (Sesamum indicum L.). Indian J. Pl. Phy. 33 (1): 94-96.

Singh, V. K.; H. G. Singh and Y. S. Chauhan. 1986. Heterosis in sesame Farm Sci. J. 1 (1-2): $65-69$.

Thiyagu, K., G. Kandasamy, N. Manivannan and V. Muralidharan. 2007a. Studies on heterosis in genetically diverse lines of cultivated sesame (Sesamum indicum L.) Madras Agric. J. 94 (7-12): 162 - 167.

Toprope, V. N. 2009. Heterosis and inbreeding depression in sesame. Journal of Maharashtra Agricultural Universities, 34(2): 220- 222.

\section{How to cite this article:}

Karande, G.R., S.V. Yamgar, A.A. Waghmode and Wadikar, P.B. 2018. Exploitation of Heterosis for Yield and Yield Contributing Character in Sesame (Sesamum indicum L.). Int.J.Curr.Microbiol.App.Sci. 7(02): 299-308. doi: https://doi.org/10.20546/ijcmas.2018.702.039 